\title{
REGULARITY AND GROWTH CONDITIONS FOR FAST ESCAPING POINTS OF ENTIRE FUNCTIONS
}

\author{
Vasiliki Evdoridou \\ The Open University, Department of Mathematics and Statistics \\ Walton Hall, Milton Keynes MK7 6AA, U.K.; vasiliki.evdoridou@open.ac.uk
}

\begin{abstract}
Let $f$ be a transcendental entire function. The fast escaping set $A(f)$ plays a key role in transcendental dynamics and so it is useful to be able to identify points in this set. Recently it was shown that, under certain conditions, the quite fast escaping set, $Q(f)$, and the related set $Q_{2}(f)$, are equal to $A(f)$. In this paper we generalise these sets by introducing a family of sets $Q_{m}(f), m \in \mathbf{N}$, and give several conditions under which $Q_{m}(f)$ is equal to $A(f)$.
\end{abstract}

\section{Introduction}

Let $f$ be a transcendental entire function. The set of points $z \in \mathbf{C}$ for which $\left(f^{n}\right)_{n \in \mathbf{N}}$ forms a normal family in some neighbourhood of $z$ is called the Fatou set $F(f)$ and the complement of $F(f)$ is the Julia set $J(f)$. An introduction to the properties of these sets can be found in [2].

A lot of work has been done in recent years on a conjecture of Eremenko on the escaping set of $f$. The escaping set $I(f)$ of $f$ is defined as follows:

$$
I(f)=\left\{z \in \mathbf{C}: f^{n}(z) \rightarrow \infty\right\}
$$

and it was first studied by Eremenko in [8] who showed that for any transcendental entire function $f$, we have $I(f) \cap J(f) \neq \emptyset, J(f)=\partial I(f)$ and all the components of $\overline{I(f)}$ are unbounded. His conjecture, that all the components of $I(f)$ are unbounded, is still an open question. Significant progress has been made on the conjecture by Rippon and Stallard who proved that $I(f)$ has at least one unbounded component (see [14, Theorem 1]). In order to do this, they considered a subset of the escaping set known as the fast escaping set, $A(f)$. This set was introduced by Bergweiler and Hinkkanen in [3]. We will use the definition given by Rippon and Stallard in [16] according to which

$$
A(f)=\left\{z \text { : there exists } \ell \in \mathbf{N} \text { such that }\left|f^{n+\ell}(z)\right| \geq M^{n}(R, f), \text { for } n \in \mathbf{N}\right\},
$$

where

$$
M(r, f)=M(r)=\max _{|z|=r}|f(z)|, \quad \text { for } r>0,
$$

and $R>0$ is large enough to ensure that $M(r)>r$ for $r \geq R$. In the same paper they showed that $A(f)$ has properties similar to the properties of $I(f)$ listed above. (Some of these results were shown in [3].) Note that the definition of $A(f)$ is independent of the choice of $R>0$ with the property that $M(r)>r$ for $r \geq R$ (see [16, Theorem 2.2(b)]).

The set $A(f)$ also has other nice properties (described in [16]) and plays a key role in iteration of transcendental entire functions and, more widely, in the iteration

https://doi.org/10.5186/aasfm.2017.4258

2010 Mathematics Subject Classification: Primary 37F10; Secondary 30D05.

Key words: Entire function, fast escaping set, quite fast escaping set, regularity, finite order, positive lower order. 
of quasiregular mappings and transcendental self-maps of the punctured plane (see [6] and [11]). Thus it is useful to be able to identify points that are fast escaping. In [16, Theorem 2.7], it is shown that points that eventually escape faster than the iterates of the function $\mu_{\varepsilon}$ defined by $\mu_{\varepsilon}(r)=\varepsilon M(r), \varepsilon \in(0,1), r>0$, are actually fast escaping.

It is natural to ask whether this $\mu_{\varepsilon}$ can be replaced by a smaller function. In this context, Rippon and Stallard introduced the quite fast escaping set $Q(f)$ in [17], which is defined as follows:

$$
Q(f)=\left\{z: \exists \varepsilon \in(0,1), \ell \in \mathbf{N} \text { such that }\left|f^{n+\ell}(z)\right| \geq \mu_{\varepsilon}^{n}(R), \text { for } n \in \mathbf{N}\right\},
$$

where $\mu_{\varepsilon}=M(r)^{\varepsilon}, \varepsilon \in(0,1)$, and $R>0$ is such that $\mu_{\varepsilon}(r)>r$ for $r \geq R$.

Points that are quite fast escaping arise naturally in complex dynamics and had been used earlier by Bergweiler, Karpińska and Stallard in [5] and by Peter in [12] in results on the Hausdorff measure and Hausdorff dimension of $I(f)$ and $J(f)$. It was shown in [17] that there are many classes of functions for which $Q(f)=A(f)$.

In [10] we generalised the quite fast escaping set by introducing the following family of larger sets:

$$
Q_{m}(f)=\left\{z: \exists \varepsilon \in(0,1), \ell \in \mathbf{N} \text { such that }\left|f^{n+\ell}(z)\right| \geq \mu_{m, \varepsilon}^{n}(R), \text { for } n \in \mathbf{N}\right\},
$$

where $\mu_{m, \varepsilon}$ is defined by

$$
\log ^{m} \mu_{m, \varepsilon}(r)=\varepsilon \log ^{m} M(r), m \in \mathbf{N}, \varepsilon \in(0,1),
$$

whenever the right-hand side of the equality is well defined, and whenever there exists $R=R(f, \varepsilon)>0$ such that $\mu_{m, \varepsilon}(r)>r$ for $r \geq R$. In particular, whenever $Q_{m}(f)$ is defined we have $Q_{m}(f) \subset I(f)$. For $m=1$ there always exists $R>0$ such that $\mu_{1, \varepsilon}(r)>r$ for $r \geq R$. In the case where $m=1$ we obtain the quite fast escaping set $Q(f)$; that is, $Q_{1}(f)=Q(f)$. The definition of $Q_{m}(f)$ is independent of the choice of $R$ (as for $A(f)$ and $Q(f)$ ).

Note that for $0<\varepsilon<1$ we have $\mu_{m, \varepsilon}(r)<\mu_{1, \varepsilon}(r)<M(r)$, for any $m \geq 2$ and for $r$ large enough, so

$$
A(f) \subset Q(f) \subset Q_{m}(f) \subset I(f) .
$$

In Section 2 we give a large class of functions for which $\mu_{m, \varepsilon}(r)$ is greater than $r$ for $r$ large enough.

Our main goal is to find classes of functions for which $Q_{m}(f)=A(f)$ for all $m \in \mathbf{N}$ since $A(f)$ plays a significant role and the conditions for a point to be in $Q_{m}(f)$ could be easier to check than the conditions for a point to be in $A(f)$. In [10] we considered the case $m=2$, that is,

$$
\mu_{2, \varepsilon}(r)=\exp \left((\log M(r))^{\varepsilon}\right)
$$

and we found regularity conditions that imply that $Q_{2}(f)=A(f)$. In particular, we proved that any transcendental entire function of finite order and positive lower order satisfies $Q_{2}(f)=A(f)$. Recall that the order $\rho(f)$ and lower order $\lambda(f)$ of $f$ are defined by

$$
\rho(f)=\limsup _{r \rightarrow \infty} \frac{\log \log M(r)}{\log r}, \quad \lambda(f)=\liminf _{r \rightarrow \infty} \frac{\log \log M(r)}{\log r} .
$$

In this paper we introduce new techniques that enable us to generalise this result for any $m \in \mathbf{N}$ as given in the following theorem:

Theorem 1.1. Let $f$ be a transcendental entire function of finite order and positive lower order. Then $Q_{m}(f)$ is defined and $Q_{m}(f)=A(f), m \in \mathbf{N}$. 
In particular, Theorem 1.1 implies that functions in the Eremenko-Lyubich class $\mathcal{B}$ that have finite order satisfy $Q_{m}(f)=A(f)$. Indeed, functions in the class $\mathcal{B}$ have positive lower order and in fact have lower order not less than $1 / 2$ (see [13, Lemma 3.5]). Note that the class $\mathcal{B}$ consists of transcendental entire functions whose set of singular values (that is, critical values and asymptotic values; see [9]) is bounded, and it is much studied in complex dynamics. Classes of functions that satisfy the hypothesis of Theorem 1.1 were studied, for example, in [4] and [19].

We prove the theorem in two different ways. The first proof is based on a new regularity condition and the second on a growth condition. In Section 3 we give our first proof of Theorem 1.1 which is in two steps. We first introduce a new regularity condition which we call $m$-log-regularity and that implies that $Q_{m}(f)=A(f)$. Let $f$ be a transcendental entire function. Then $f$ is $m$-log-regular if and only if, for any $\varepsilon \in(0,1)$, there exist $R>0$ and $k>1$ such that

$$
\mu_{m, \varepsilon}\left(\exp ^{m-1}\left(r^{k}\right)\right) \geq \exp ^{m-1}\left(M(r)^{k}\right), \quad \text { for } r \geq R \text {. }
$$

We are not aware whether there is any relationship between the different $m$-logregularity conditions. For $m=1$ we obtain the log-regularity condition that was first introduced by Anderson and Hinkkanen in [1] and was used by Rippon and Stallard in [17] as a sufficient condition for $Q(f)$ to be equal to $A(f)$. We then show that any function that is $m$-log-regular satisfies $Q_{m}(f)=A(f)$. In the second step, we prove that all functions of finite order and positive lower order are $m$-log-regular.

In Section 4 we prove Theorem 1.1 in a different way, again in two steps. We give a growth condition that is sufficient for $Q_{m}(f)=A(f)$ and then we show that any transcendental entire function of finite order and positive lower order satisfies this growth condition.

In [10] we introduced a regularity condition called strong log-regularity which implies that $Q_{2}(f)=A(f)$. In Section 5 we show how strong log-regularity is related to 2-log-regularity. In particular we prove that a strongly log-regular function of finite order is always 2-log-regular and we give an example of a 2-log-regular function of finite order that fails to be strongly log-regular.

Acknowledgments. I would like to thank my supervisors Prof. Phil Rippon and Prof. Gwyneth Stallard for their help in the preparation of this paper and Dave Sixsmith for helpful comments.

\section{Properties of $Q_{m}(f)$}

In this section we prove some basic properties of $Q_{m}(f)$. Just as for $\mu_{2, \varepsilon}$, in the general case we do not know a priori that, for any given transcendental entire function, $\mu_{m, \varepsilon}(r)$ is greater than $r$ for $r$ large enough. We show first that, for a large class of functions, there is always a positive $R$ such that $\mu_{m, \varepsilon}(r)>r$, for $r \geq R$, and hence for these functions $Q_{m}(f)$ is defined.

Theorem 2.1. Let $f$ be a transcendental entire function, $m \geq 2$ and $\varepsilon \in(0,1)$. If there exist $q>0, r_{0}>0$ and $n \in \mathbf{N}$ such that

$$
M(r) \geq \exp ^{n+1}\left(\left(\log ^{n} r\right)^{q}\right), \quad \text { for } r \geq r_{0},
$$

then, for any $c>1$, there exists $R>0$ such that

$$
\mu_{m, \varepsilon}(r)>c r, \quad \text { for } r \geq R \text {. }
$$

Note that (2.1) is true for all functions of positive lower order as well as some functions of zero lower order. In particular, it is true for all the functions in class $\mathcal{B}$ 
as they have lower order not less than $1 / 2$. In order to prove Theorem 2.1 we use the following inequality.

Lemma 2.2. For any $n \in \mathbf{N}, p \geq 1$ and $a_{1}, \ldots, a_{n}, b_{1}, \ldots, b_{n}>0$ there exists $R>0$ such that

$$
a_{1} \log \left(a_{2} \log \ldots \log \left(a_{n} r\right) \ldots\right) \geq \log \left(b_{1} \log \ldots \log \left(\left(b_{n} r\right)^{p}\right) \ldots\right), \quad \text { for } r \geq R .
$$

Proof. It suffices to prove (2.2) for $p>1$. We use proof by induction. As $a_{1} r \geq \log \left(\left(b_{1} r\right)^{p}\right)$, for $r$ large enough, (2.2) is certainly true for $n=1$. Suppose now that (2.2) is true for some $n \geq 1$. We will deduce that

$(2.3) \quad a_{1} \log \left(a_{2} \log \ldots \log \left(a_{n} \log \left(a_{n+1} r\right)\right) \ldots\right) \geq \log \left(b_{1} \log \ldots \log \left(b_{n} \log \left(\left(b_{n+1} r\right)^{p}\right)\right) \ldots\right)$,

for $r$ large enough. To do this, note first that, for $r$ large enough,

$$
a_{1} \log \left(a_{2} \log \ldots \log \left(a_{n} \log \left(a_{n+1} r\right)\right) \ldots\right) \geq \log \left(b_{1} \log \ldots \log \left(\left(b_{n} \log \left(a_{n+1} r\right)\right)^{p}\right) \ldots\right),
$$

by (2.2). Then, in order to deduce (2.3) it suffices to show that

$$
\left(b_{n} \log \left(a_{n+1} r\right)\right)^{p} \geq b_{n} p \log \left(b_{n+1} r\right),
$$

for $r$ large enough. Note now that (2.4) is true since there exists $R>0$ such that

$$
\frac{b_{n}^{p-1}}{p}\left(\log \left(a_{n+1} r\right)\right)^{p} \geq \log \left(b_{n+1} r\right), \text { for } r \geq R,
$$

and the result follows.

Proof of Theorem 2.1. By definition, $\mu_{m, \varepsilon}(r)=\exp ^{m}\left(\varepsilon \log ^{m} M(r)\right)$, so we have to show that

$$
\exp ^{m}\left(\varepsilon \log ^{m} M(r)\right)>c r, \quad \text { for } r \text { large enough. }
$$

We consider three different cases depending on the relative sizes of $m$ and the positive integer $n$ from (2.1).

a) Suppose that $n+1=m$. Then, by $(2.1)$,

$$
\begin{aligned}
\exp ^{m}\left(\varepsilon \log ^{m} M(r)\right) & \geq \exp ^{m}\left(\varepsilon \log ^{m}\left(\exp ^{n+1}\left(\left(\log ^{n} r\right)^{q}\right)\right)\right) \\
& =\exp ^{m}\left(\varepsilon\left(\log ^{n} r\right)^{q}\right) \\
& >c r, \quad \text { for } r \text { large enough, }
\end{aligned}
$$

since $\varepsilon\left(\log ^{n} r\right)^{q}>\log \log ^{n} c r=\log ^{m} c r$, for $r$ large enough.

b) Suppose that $n+1<m$. Then, by $(2.1)$,

$\exp ^{m}\left(\varepsilon \log ^{m} M(r)\right) \geq \exp ^{m}\left(\varepsilon \log ^{m}\left(\exp ^{n+1}\left(\left(\log ^{n} r\right)^{q}\right)\right)\right)=\exp ^{m}\left(\varepsilon \log ^{m-n-1}\left(\left(\log ^{n} r\right)^{q}\right)\right)$.

Hence, we need to show that, for any $c>1$,

$$
\exp ^{m}\left(\varepsilon \log ^{m-n-1}\left(\left(\log ^{n} r\right)^{q}\right)\right)>c r, \quad \text { for } r \text { large enough, }
$$

or, equivalently,

$$
\varepsilon \log ^{m-n-1}\left(\left(\log ^{n} r\right)^{q}\right)>\log ^{m} c r, \quad \text { for } r \text { large enough, }
$$

which holds by applying Lemma 2.2 with $n$ replaced by $m, p=1, a_{1}=\varepsilon, a_{m-n-1}=$ $q, b_{m}=c$ and all the other coefficients equal to 1 .

c) Finally, suppose that $n+1>m$. Then, by (2.1),

$\exp ^{m}\left(\varepsilon \log ^{m} M(r)\right) \geq \exp ^{m}\left(\varepsilon \log ^{m}\left(\exp ^{n+1}\left(\left(\log ^{n} r\right)^{q}\right)\right)\right)=\exp ^{m}\left(\varepsilon \exp ^{n+1-m}\left(\left(\log ^{n} r\right)^{q}\right)\right)$.

Hence, we need to show that, for any $c>1$,

$$
\exp ^{m}\left(\varepsilon \exp ^{n+1-m}\left(\left(\log ^{n} r\right)^{q}\right)\right)>c r, \quad \text { for } r \text { large enough, }
$$


or, equivalently,

$$
\varepsilon \exp ^{n+1-m}\left(\left(\log ^{n} r\right)^{q}\right)>\log ^{m} c r, \quad \text { for } r \text { large enough. }
$$

Note now that $(2.8)$ is equivalent to

$$
\left(\log ^{n} r\right)^{q}>\log ^{n+1-m}\left(\frac{1}{\varepsilon} \log ^{m} c r\right), \quad \text { for } r \text { large enough. }
$$

If we apply Lemma 2.2 with $n$ replaced by $n+2, p=1, a_{1}=q, b_{n+2-m}=1 / \varepsilon, b_{n+2}=c$ and the rest of the coefficients equal to 1 we obtain

$$
q \log ^{n+1} r>\log ^{n+2-m}\left(\frac{1}{\varepsilon} \log ^{m} c r\right),
$$

for $r$ large enough and so (2.9) follows.

We now show that $Q_{m}(f)$ has some basic properties similar to those of $I(f), A(f)$ and $Q(f)$.

Theorem 2.3. Let $f$ be a transcendental entire function and $m \in \mathbf{N}$ be such that $Q_{m}(f)$ is defined. Then

$$
Q_{m}(f) \neq \emptyset, \quad Q_{m}(f) \cap J(f) \neq \emptyset, \quad \text { and } \quad J(f)=\overline{Q_{m}(f) \cap J(f)} .
$$

If, in addition, for any $c>1$ and $\varepsilon \in(0,1)$, there exists $R>0$ such that

$$
\mu_{m, \varepsilon}(r)>c r, \quad \text { for } r \geq R,
$$

then

$$
J(f)=\partial Q_{m}(f),
$$

and $\overline{Q_{m}(f)}$ has no bounded components.

Proof. All the properties above hold for $A(f)$ (see [16]). As $A(f) \subset Q_{m}(f)$, we certainly have $Q_{m}(f) \neq \emptyset$ and $Q_{m}(f) \cap J(f) \neq \emptyset$. Also $J(f)=\overline{A(f) \cap J(f)} \subset$ $\overline{Q_{m}(f) \cap J(f)}$. Since $J(f)$ is closed, we also have $\overline{Q_{m}(f) \cap J(f)} \subset J(f)$ and so the third property is also true.

In order to prove the two remaining properties, we follow the arguments in the proof of [17, Theorem 2.1]. Note first that $Q_{m}(f)$ is infinite and completely invariant under $f$ which, since $J(f)$ is the smallest closed completely invariant set with at least three points, implies that $J(f) \subset \overline{Q_{m}(f)}$. But any open subset of $Q_{m}(f)$ is contained in $F(f)$ since it contains no periodic points of $f$, and so $J(f) \subset \partial Q_{m}(f)$.

Suppose now that $\partial Q_{m}(f) \cap U \neq \emptyset$, where $U$ is a Fatou component and take $z \in \partial Q_{m}(f) \cap U$. Take also a disc $\Delta$ centred at $z$ with $\bar{\Delta} \subset U$. Then $\Delta \cap Q_{m}(f) \neq \emptyset$ and we take $z_{0} \in Q_{m}(f) \cap U$. If $U$ is simply connected then, by applying [2, Lemma 7], we have that there exists $C>0$ such that

$$
\left|f^{n}\left(z_{1}\right)\right| \geq C\left|f^{n}\left(z_{0}\right)\right|,
$$

for any $z_{1} \in \Delta$ and $n \in \mathbf{N}$. If $(2.10)$ holds there exist $R>0$ and $\varepsilon \in(0,1)$ such that $\mu_{m, \varepsilon}(r)>r, r \geq R$ and $\ell \in \mathbf{N}$ such that, for $n \in \mathbf{N}$,

$$
\left|f^{n+\ell}\left(z_{1}\right)\right| \geq C\left|f^{n+\ell}\left(z_{0}\right)\right| \geq C \mu_{m, \varepsilon}^{n}(R),
$$

and so

$$
\left|f^{n+\ell}\left(z_{1}\right)\right| \geq \mu_{m, \varepsilon}^{n-1}(R),
$$

by (2.10). Therefore, any point in the neighbourhood $\Delta$ of $z$ lies in $Q_{m}(f)$, which gives a contradiction. If the Fatou component $U$ is multiply connected then $U \subset$ 
$A(f) \subset Q_{m}(f)$ (see [14, Theorem 2]) and so there is again a contradiction. Hence, $\partial Q_{m}(f) \subset J(f)$.

Finally, if $\overline{Q_{m}(f)}$ has a bounded component, $E$ say, then there is an open topological annulus $A$ lying in the complement of $\overline{Q_{m}(f)}$ that surrounds $E$. Since $\overline{Q_{m}(f)}$ is completely invariant under $f, A$ is contained in $F(f)$ by Montel's theorem. But from the previous property, $J(f)=\partial Q_{m}(f)$ and so $A$ is contained in a multiply connected Fatou component. As any multiply connected Fatou component is contained in $A(f) \subset Q_{m}(f)$ we deduce that $A \subset Q_{m}(f)$ which gives a contradiction.

\section{Regularity conditions for $Q_{m}(f)=A(f)$}

In this section, we use regularity conditions to prove Theorem 1.1. In the introduction we defined $m$-log-regularity, which is a sufficient condition for $Q_{m}(f)$ to be equal to $A(f)$. In fact, there also exists another regularity condition called $m$-weak-regularity that is equivalent to $Q_{m}(f)=A(f)$. We will show later that $m$ log-regularity is stronger than $m$-weak-regularity and hence if $f$ is $m$-log-regular then $Q_{m}(f)=A(f)$. Finally, we will use these ideas in order to prove Theorem 1.1. Note that $m$-log-regularity is easier to check than $m$-weak-regularity which is defined as follows:

Let $R>0$ be any value such that $M(r)>r$ for $r \geq R$. We say that $f$ is $m$-weakly-regular if for any $\varepsilon \in(0,1)$ there exists $r=r(R)>0$ such that

$$
\mu_{m, \varepsilon}^{n}(r) \geq M^{n}(R), \quad \text { for } n \in \mathbf{N},
$$

or, equivalently, if there exists $\ell=\ell(R) \in \mathbf{N}$ such that

$$
\mu_{m, \varepsilon}^{n+\ell}(R) \geq M^{n}(R), \quad \text { for } n \in \mathbf{N} .
$$

For $m=1$ we have the weak regularity that was introduced by Rippon and Stallard in [17].

We will show that $m$-weak-regularity is a necessary and sufficient condition for $f$ to satisfy $Q_{m}(f)=A(f)$. In order to prove our result we make use of the following theorem of Rippon and Stallard (see [18, Theorem 1.4] and [17, Theorem 3.1]).

Theorem 3.1. Let $f$ be a transcendental entire function. There exists $R=$ $R(f)>0$ with the property that whenever $\left(a_{n}\right)$ is a positive sequence such that

$$
a_{n} \geq R \text { and } a_{n+1} \leq M\left(a_{n}\right), \quad \text { for } n \in \mathbf{N},
$$

there exists a point $\zeta \in J(f)$ and a sequence $\left(n_{j}\right)$ with $n_{j} \rightarrow \infty$ such that

$$
\left|f^{n}(\zeta)\right| \geq a_{n}, \quad \text { for } n \in \mathbf{N}, \quad \text { but }\left|f^{n_{j}}(\zeta)\right| \leq M^{2}\left(a_{n_{j}}\right), \quad \text { for } j \in \mathbf{N} \text {. }
$$

We now prove our result. The proof uses arguments used in the proof of [17, Theorem 1.2].

Theorem 3.2. Let $f$ be a transcendental entire function. Then $f$ is $m$-weaklyregular if and only if $Q_{m}(f)$ is defined and $Q_{m}(f)=A(f)$.

Proof. Suppose that $f$ is $m$-weakly-regular and let $R>0$ be such that $M(r)>r$ for $r \geq R$. Then there exists $r=r(R)>0$ such that

$$
\mu_{m, \varepsilon}^{n}(r) \geq M^{n}(R), \quad \text { for } n \in \mathbf{N},
$$

and so $Q_{m}(f)$ is defined.

If $z \in Q_{m}(f)$, then there exist $\varepsilon \in(0,1)$ and $\ell \in \mathbf{N}$ such that

$$
\left|f^{n+\ell}(z)\right| \geq \mu_{m, \varepsilon}^{n}(R), \quad \text { for } n \in \mathbf{N} \text {. }
$$


Let $r=r(R)$ be as above. Then there exists $N \in \mathbf{N}$ such that $\mu_{m, \varepsilon}^{N}(R)>r$ so

$$
\left|f^{n+\ell+N}(z)\right| \geq \mu_{m, \varepsilon}^{n+N}(R) \geq \mu_{m, \varepsilon}^{n}(r) \geq M^{n}(R), \quad \text { for } n \in \mathbf{N},
$$

and hence $z \in A(f)$. Thus $Q_{m}(f) \subset A(f)$. Clearly $A(f) \subset Q_{m}(f)$ and so we have $Q_{m}(f)=A(f)$ as claimed.

In order to show that the opposite direction of the theorem is also true we will prove that if $f$ is not $m$-weakly-regular and $Q_{m}(f)$ is defined then $Q_{m}(f) \backslash A(f)$ is nonempty. Take $R>0$ such that $\mu_{m, \varepsilon}(r)>r$, for $r \geq R$. Since $f$ is not $m$-weakly-regular, for any $\ell \in \mathbf{N}$ there exist $\varepsilon \in(0,1)$ and $n(\ell) \in \mathbf{N}$ such that $\mu_{m, \varepsilon}^{n(\ell)+\ell}(R)<M^{n(\ell)}(R)$ and hence, for any $n \in \mathbf{N}$ with $n>n(\ell)$, we have

$$
\mu_{m, \varepsilon}^{n+\ell}(R)<M^{n}(R)
$$

Now, by Theorem 3.1, with $a_{n}=\mu_{m, \varepsilon}^{n}(R), n \in \mathbf{N}$, there exists a point $\zeta$ and a sequence $\left(n_{j}\right) \rightarrow \infty$ as $j \rightarrow \infty$, such that

$$
\left|f^{n}(\zeta)\right| \geq \mu_{m, \varepsilon}^{n}(R), \quad \text { for } n \in \mathbf{N}
$$

and

$$
\left|f^{n_{j}}(\zeta)\right| \leq M^{2}\left(\mu_{m, \varepsilon}^{n_{j}}(R)\right), \quad \text { for } j \in \mathbf{N} .
$$

It follows from (3.4) that $\zeta \in Q_{m}(f)$. Also, (3.3) and (3.5) together imply that, for each $\ell \in \mathbf{N}$ and sufficiently large values of $j$, we have

$$
\left|f^{\left(n_{j}-\ell+2\right)+\ell-2}(\zeta)\right|=\left|f^{n_{j}}(\zeta)\right| \leq M^{2}\left(\mu_{m, \varepsilon}^{n_{j}}(R)\right)<M^{2}\left(M^{n_{j}-\ell}(R)\right)=M^{n_{j}-\ell+2}(R) .
$$

Hence, $\zeta \notin A(f)$, so $Q_{m}(f) \neq A(f)$, as required.

We now give the proof of Theorem 1.1. The proof is in two steps. First, we prove the following result which implies that all $m$-log-regular functions satisfy $Q_{m}(f)=$ $A(f)$.

Theorem 3.3. Let $f$ be a transcendental entire function. If $f$ is $m$-log-regular, then $f$ is $m$-weakly-regular and hence $Q_{m}(f)=A(f)$.

Proof. Suppose that $f$ is $m$-log-regular and let $0<\varepsilon<1$. Let $R>0$ be so large that $M(r)>r$ for $r \geq R$. Since $f$ is $m$-log-regular, for any $\varepsilon \in(0,1)$ there exists $r_{0} \geq R$ and $k>1$ such that

$$
\mu_{m, \varepsilon}\left(\exp ^{m-1}\left(r^{k}\right)\right) \geq \exp ^{m-1}\left(M(r)^{k}\right), \quad \text { for } r \geq r_{0} .
$$

Hence,

$$
\mu_{m, \varepsilon}\left(\mu_{m, \varepsilon}\left(\exp ^{m-1}\left(r^{k}\right)\right)\right) \geq \mu_{m, \varepsilon}\left(\exp ^{m-1}\left(M(r)^{k}\right)\right) \geq \exp ^{m-1}\left(\left(M(M(r))^{k}\right)\right.
$$

and so, using this argument repeatedly, we have

$$
\mu_{m, \varepsilon}^{n}\left(\exp ^{m-1}\left(r^{k}\right)\right) \geq \exp ^{m-1}\left(M^{n}(r)^{k}\right), \quad \text { for } \geq r_{0} \quad \text { and } \quad n \in \mathbf{N} .
$$

Thus, whenever $r \geq r_{0}$, we have

$$
\mu_{m, \varepsilon}^{n}\left(\exp ^{m-1}\left(r^{k}\right)\right) \geq M^{n}(r) \geq M^{n}(R), \quad \text { for } n \in \mathbf{N},
$$

and so $f$ is $m$-weakly-regular. Hence, by Theorem $3.2, Q_{m}(f)=A(f)$.

The second part of the proof of Theorem 1.1 is to show that all functions of finite order and positive lower order are $m$-log-regular. In order to prove this we will need the following lemma. 
Lemma 3.4. For any $n \in \mathbf{N}$ and any $d>0, q, q^{\prime} \in(0,1)$, there exists $R>0$ such that

$$
\log ^{n}\left(r^{q}\right)>d\left(\log ^{n} r\right)^{q^{\prime}}, \quad \text { for } r \geq R
$$

Proof. We will prove (3.6) using induction. For $n=1$,

$$
q \log r>d(\log r)^{q^{\prime}}, \quad \text { for } r \text { large enough. }
$$

Suppose that (3.6) is true for some $n \in \mathbf{N}$. Then

$$
d\left(\log ^{n+1} r\right)^{q^{\prime}}=d\left(\log ^{n}(\log r)\right)^{q^{\prime}}<\log ^{n}\left((\log r)^{q}\right), \quad \text { for } r \text { large enough. }
$$

Hence, in order to prove (3.6) it suffices to show that there exists $R>0$ such that

$$
\log ^{n+1}\left(r^{q}\right)>\log ^{n}\left((\log r)^{q}\right), \quad \text { for } r \geq R, n \in \mathbf{N},
$$

or equivalently that

$$
\log \left(r^{q}\right)>(\log r)^{q}, \quad \text { for } r \geq R,
$$

which is true, and so, the result follows.

We now prove the following result.

Theorem 3.5. Let $f$ be a transcendental entire function of finite order and positive lower order. Then $f$ is $m$-log-regular.

Proof. Let $f$ be a transcendental entire function of finite order and positive lower order. We begin by noting that there exist $0<q<p$ such that

$$
e^{r^{q}} \leq M(r) \leq e^{r^{p}}, \text { for } r \text { large enough. }
$$

By the definition of $\mu_{m, \varepsilon}$, in order to prove that $f$ is $m$-log-regular we need to show that for any $\varepsilon \in(0,1)$, there exist $R>0$ and $k>1$ such that $f$ satisfies (1.2) or, equivalently,

$$
\varepsilon \log ^{m} M\left(\exp ^{m-1}\left(r^{k}\right)\right) \geq \log ^{m}\left(\exp ^{m-1}\left(M(r)^{k}\right)\right), \quad \text { for } r \geq R .
$$

But (3.7) implies that

$$
\log ^{m} M\left(\exp ^{m-1}\left(r^{k}\right)\right) \geq \log ^{m-1}\left(\left(\exp ^{m-1}\left(r^{k}\right)\right)^{q}\right)
$$

and

$$
\log ^{m}\left(\exp ^{m-1}\left(M(r)^{k}\right)\right) \leq \log ^{m}\left(\exp ^{m}\left(k r^{p}\right)\right)=k r^{p},
$$

and so (3.8) is implied by

$$
\varepsilon \log ^{m-1}\left(\left(\exp ^{m-1}\left(r^{k}\right)\right)^{q}\right) \geq k r^{p},
$$

that is,

$$
\left(\exp ^{m-1}\left(r^{k}\right)\right)^{q} \geq \exp ^{m-1}\left(\frac{k r^{p}}{\varepsilon}\right), \quad \text { for } r \text { large enough. }
$$

We set $r^{k}=\log ^{m-1} s$ and (3.9) becomes

$$
\log ^{m-1}\left(s^{q}\right) \geq \frac{k}{\varepsilon}\left(\log ^{m-1} s\right)^{p / k}, \quad \text { for } s \text { large enough. }
$$

For $m=1$, if we choose $k>p / q$ then, for any $\varepsilon \in(0,1)$, (3.10) holds for $s$ large enough. For $m>1$, if we choose $k>p$ then, for any $\varepsilon \in(0,1),(3.10)$ holds for $s$ large enough, by Lemma 3.4.

It is easy to see that if we combine Theorem 3.3 and Theorem 3.5 we obtain Theorem 1.1. 


\section{Growth conditions for $Q_{m}(f)=A(f)$}

In this section we give a second proof of Theorem 1.1 by introducing a growth condition, given in the following theorem, that implies that $Q_{m}(f)=A(f)$. The same lower bound for $m=2$ appears in [15, Theorem 6].

Theorem 4.1. Let $f$ be a transcendental entire function, $m \geq 2$ and $\phi_{m}(t)=$ $\log ^{m-1} M\left(\exp ^{m-1}(t)\right)$. If there exist $0<q<1$ and $0<\tilde{q}<\infty$ such that, for some $n \geq 0$,

$$
\exp ^{n+m-1}\left(\left(\log ^{n+m-2} t\right)^{q}\right) \leq \phi_{m}(t) \leq \exp ^{n+m-1}\left(\left(\log ^{n+m-2} t\right)^{\tilde{q}}\right),
$$

for $t$ large enough, then

(i) for any $d>1$ and any $p>1 / q$ there exists $t_{0}>0$ such that

$$
\phi_{m}\left(\psi_{m}(t)\right) \geq\left(\psi_{m}\left(\phi_{m}(t)\right)\right)^{d}, \quad \text { for } t \geq t_{0},
$$

where $\psi_{m}(t)=\exp ^{n+m-1}\left(\left(\log ^{n+m-1} t\right)^{p}\right)$;

(ii) $f$ is $m$-weakly-regular and so $Q_{m}(f)=A(f)$.

Remark 4.1. As $0<q<1$, the left bound in (4.1) becomes smaller as $n$ increases. If we also take $\tilde{q}>1$, then the right bound increases with $n$ and hence the condition (4.1) is more easily satisfied for larger $n$. As we will prove in Theorem 4.2, all functions of positive lower order and finite order satisfy (4.1) with $n=0$.

Proof. (i) We have that

$$
\begin{aligned}
\psi_{m}\left(\phi_{m}(t)\right) & \leq \exp ^{n+m-1}\left(\left(\log ^{n+m-1}\left(\exp ^{n+m-1}\left(\log ^{n+m-2} t\right)^{\tilde{q}}\right)\right)^{p}\right) \\
& =\exp ^{n+m-1}\left(\left(\log ^{n+m-2} t\right)^{\tilde{q} p}\right)
\end{aligned}
$$

and also

$$
\begin{aligned}
\phi_{m}\left(\psi_{m}(t)\right) & \geq \exp ^{n+m-1}\left(\left(\log ^{n+m-2}\left(\exp ^{n+m-1}\left(\left(\log ^{n+m-1} t\right)^{p}\right)\right)\right)^{q}\right) \\
& =\exp ^{n+m-1}\left(\left(\exp \left(\left(\log ^{n+m-1} t\right)^{p}\right)\right)^{q}\right)=\exp ^{n+m}\left(q\left(\log ^{n+m-1} t\right)^{p}\right) \\
& \geq \exp ^{n+m}\left(\left(\log ^{n+m-1} t\right)^{p q}\right) \geq\left(\exp ^{n+m-1}\left(\left(\log ^{n+m-2} t\right)^{p \tilde{q}}\right)\right)^{d},
\end{aligned}
$$

for any $d>1$ and for $t$ large enough, since putting $w=\log ^{n+m-1} t$ gives

$$
\begin{aligned}
\frac{\exp ^{n+m-1}\left(\left(\log ^{n+m-1} t\right)^{p q}\right)}{\exp ^{n+m-2}\left(\left(\log ^{n+m-2} t\right)^{p \tilde{q}}\right)} & =\frac{\exp ^{n+m-1}\left(w^{p q}\right)}{\exp ^{n+m-2}\left(\left(e^{w}\right)^{p \tilde{q}}\right)}=\frac{\exp ^{n+m-1}\left(w^{p q}\right)}{\exp ^{n+m-2}\left(e^{p \tilde{q} w}\right)} \\
& =\frac{\exp ^{n+m-1}\left(w^{p q}\right)}{\exp ^{n+m-1}(p \tilde{q} w)} \rightarrow \infty \quad \text { as } w \rightarrow \infty
\end{aligned}
$$

and so

$$
\exp ^{n+m-1}\left(\left(\log ^{n+m-1} t\right)^{p q}\right) \geq d \exp ^{n+m-2}\left(\left(\log ^{n+m-2} t\right)^{p \tilde{q}}\right) \text {, for } t \text { large enough. }
$$

Thus

$$
\phi_{m}\left(\psi_{m}(t)\right) \geq\left(\psi_{m}\left(\phi_{m}(t)\right)\right)^{d}, \quad d>1, \quad \text { for } t \text { large enough. }
$$

(ii) Now let $\phi_{m, \varepsilon}(t)=\phi_{m}(t)^{\varepsilon}$ and note that from the definition of $\phi_{m}$,

$$
M^{\ell}(r)=\exp ^{m-1}\left(\phi_{m}^{\ell}\left(\log ^{m-1} r\right)\right) \text {. }
$$

Note also that

$$
\begin{aligned}
\mu_{m, \varepsilon}(r) & =\exp ^{m}\left(\varepsilon \log ^{m} M(r)\right)=\exp ^{m}\left(\varepsilon \log ^{m}\left(\exp ^{m-1} \phi_{m}\left(\log ^{m-1} r\right)\right)\right) \\
& =\exp ^{m}\left(\log \left(\phi_{m, \varepsilon}\left(\log ^{m-1} r\right)\right)=\exp ^{m-1}\left(\phi_{m, \varepsilon}\left(\log ^{m-1} r\right)\right),\right.
\end{aligned}
$$


and so,

$$
\mu_{m, \varepsilon}^{\ell}(r)=\exp ^{m-1}\left(\phi_{m, \varepsilon}^{\ell}\left(\log ^{m-1} r\right)\right) .
$$

Hence, in order to show that there exists $r=r(R)>0$ such that

$$
\mu_{m, \varepsilon}^{\ell}(r) \geq M^{\ell}(R), \quad \text { for } \ell \in \mathbf{N},
$$

it suffices to show that there exists $r=r(R)>0$ such that

$$
\phi_{m, \varepsilon}^{\ell}(r) \geq \phi_{m}^{\ell}(R), \quad \text { for } \ell \in \mathbf{N} .
$$

We showed in (i) that, for any $d>1, \phi_{m}\left(\psi_{m}(t)\right) \geq\left(\psi_{m}\left(\phi_{m}(t)\right)\right)^{d}$, if $t$ is sufficiently large, or, equivalently, that given $\varepsilon>0$

$$
\phi_{m, \varepsilon}\left(\psi_{m}(t)\right) \geq \psi_{m}\left(\phi_{m}(t)\right), \quad \text { for } t \text { large enough. }
$$

Therefore,

$$
\phi_{m, \varepsilon}(s) \geq \psi_{m}\left(\phi_{m}\left(\psi_{m}^{-1}(s)\right)\right), \quad \text { for } s \text { large enough. }
$$

Since $\psi_{m}(t) \geq t$, by iterating we obtain

$$
\phi_{m, \varepsilon}^{\ell}(s) \geq \psi_{m}\left(\phi_{m}^{\ell}\left(\psi_{m}^{-1}(s)\right)\right) \geq \phi_{m}^{\ell}\left(\psi_{m}^{-1}(s)\right), \quad \text { for } s \text { large enough. }
$$

The result follows.

In order to complete the proof of Theorem 1.1 it remains to show that Theorem 4.1 can be applied to functions of finite order and positive lower order.

Theorem 4.2. Let $f$ be a transcendental entire function of finite order and positive lower order. Then $f$ satisfies the hypotheses of Theorem 4.1 and hence $Q_{m}(f)=A(f)$.

Proof. As $f$ is of finite order and positive lower order, (3.7) implies that, for $m \geq 2$ there exist $q \in(0,1)$ and $p \in(1, \infty)$ such that

$$
\log ^{m-2}\left(\left(\exp ^{m-1} t\right)^{q}\right) \leq \phi_{m}(t)=\log ^{m-1} M\left(\exp ^{m-1} t\right) \leq \log ^{m-2}\left(\left(\exp ^{m-1} t\right)^{p}\right),
$$

for $t$ large enough.

In order to show that (4.2) implies (4.1), it suffices to show that

$$
\exp ^{m-1}\left(\left(\log ^{m-2} t\right)^{q}\right) \leq \log ^{m-2}\left(\left(\exp ^{m-1} t\right)^{q}\right)
$$

and

$$
\log ^{m-2}\left(\left(\exp ^{m-1} t\right)^{p}\right) \leq \exp ^{m-1}\left(\left(\log ^{m-2} t\right)^{p}\right),
$$

for $t$ large enough. Note that (4.3) is equivalent to

$$
\left(\log ^{m-2} t\right)^{q} \leq \log ^{2 m-3}\left(\exp ^{m-1} t\right)^{q}, \quad \text { for } t \text { large enough, }
$$

which, for $s=\exp ^{m-1} t$, becomes

$$
\left(\log ^{2 m-3} s\right)^{q} \leq \log ^{2 m-3} s^{q}, \quad \text { for } s \text { large enough. }
$$

By Lemma 3.4, (4.5) holds for $s$ large enough and hence so does (4.3).

Similarly, using Lemma 3.4, one can show that (4.4) is true. Therefore, the hypotheses of Theorem 4.1 are satisfied for $q$ and $p=\tilde{q}$. 


\section{2-log-regularity and strong log-regularity}

In [10] we introduced a sufficient condition for $Q_{2}(f)=A(f)$ called strong logregularity. A transcendental entire function $f$ is strongly log-regular if, for any $\varepsilon \in$ $(0,1)$, there exist $R>0$ and $k>1$ such that, for $r>R$,

$$
\log M\left(r^{k}\right) \geq(k \log M(r))^{1 / \varepsilon} .
$$

Note that strong log-regularity implies log-regularity (see [10]), which, as mentioned in the introduction, is the same as 1-log-regularity.

Both strong log-regularity and 2-log-regularity imply $Q_{2}(f)=A(f)$ and also any transcendental entire function of finite order and positive lower order is both strongly log-regular and 2-log-regular. Therefore it is of interest to know how these two conditions are related. For a function of finite order we have the following result.

Theorem 5.1. Let $f$ be a transcendental entire function of finite order. If $f$ is strongly log-regular then $f$ is 2-log-regular.

Proof. As $f$ is of finite order, (3.7) implies that there exists $p \geq 0$, such that

$$
\log M(r) \leq r^{p}, \quad \text { for } r \text { large enough. }
$$

Also since $f$ is strongly log-regular, for any $\varepsilon \in(0,1)$, there exist $R>0$ and $k>1$ such that

$$
\log M\left(r^{k}\right) \geq(k \log M(r))^{1 / \varepsilon}, \quad \text { for } r>R .
$$

In order to show that $f$ is 2 -log-regular we will show that, for any $\varepsilon \in(0,1)$,

$$
\mu_{2, \varepsilon}\left(\exp \left(r^{k}\right)\right) \geq \exp \left(M(r)^{k}\right), \quad \text { for } r \text { large enough; }
$$

that is, using the definition of $\mu_{2, \varepsilon}(r)$,

$$
\left(\log M\left(\exp \left(r^{k}\right)\right)\right)^{\varepsilon} \geq M(r)^{k}, \quad \text { for } r \text { large enough. }
$$

It is obvious from the definition of 2-log-regularity that if the condition holds for any $\varepsilon \in\left(0,1 / e^{p}\right)$ it will hold for any $\varepsilon \in(0,1)$ and so we now fix $\varepsilon \in\left(0,1 / e^{p}\right)$ and show that (5.4) holds for this value of $\varepsilon$.

Consider now

$$
n=\left[\frac{k \log r-\log \log r}{\log k}\right],
$$

where $[x]$ denotes the integer part of the real number $x$. Then $k^{n} \leq r^{k} / \log r$, which gives us that $\exp \left(r^{k}\right) \geq r^{k^{n}}$. Hence

$$
\left(\log M\left(\exp \left(r^{k}\right)\right)\right)^{\varepsilon} \geq\left(\log M\left(r^{k^{n}}\right)\right)^{\varepsilon},
$$

and by applying (5.3) $n$ times, we deduce that

$$
\left(\log M\left(\exp \left(r^{k}\right)\right)\right)^{\varepsilon} \geq k^{1+1 / \varepsilon+\ldots+1 / \varepsilon^{n-1}}(\log M(r))^{1 / \varepsilon^{n-1}}, \quad \text { for } r \text { large enough. }
$$

Therefore, it suffices to show that

$$
(\log M(r))^{1 / \varepsilon^{n-1}} \geq M(r)^{k},
$$

or, equivalently, that

$$
\left(\frac{1}{\varepsilon}\right)^{n-1} \log \log M(r) \geq k \log M(r), \quad \text { for } r \text { large enough. }
$$


By (5.2) it is sufficient to show that

$$
\left(\frac{1}{\varepsilon}\right)^{n-1} \geq k r^{p}
$$

or, equivalently,

$$
(n-1) \log \frac{1}{\varepsilon} \geq \log k+p \log r, \quad \text { for } r \text { large enough. }
$$

In order to show that (5.6) is true we first note that it follows from (5.5) that

$$
n-1 \geq \frac{k \log r}{\log k}-\frac{\log \log r+2 \log k}{\log k},
$$

and so

$$
(n-1) \log \frac{1}{\varepsilon}-p \log r \geq\left(\left(\log \frac{1}{\varepsilon}\right) \frac{k}{\log k}-p\right) \log r-\left(\log \frac{1}{\varepsilon}\right) \frac{\log \log r+2 \log k}{\log k} .
$$

Since $\log \frac{1}{\varepsilon}>p$, there exists $R_{0}>0$ such that

$$
\left(\left(\log \frac{1}{\varepsilon}\right) \frac{k}{\log k}-p\right) \log r \geq\left(\log \frac{1}{\varepsilon}\right) \frac{\log \log r+2 \log k}{\log k}+\log k, \quad \text { for } r \geq R_{0} .
$$

Together with (5.7), this is sufficient to prove (5.6).

The converse of Theorem 5.1 is not always true though. We now use a function, that was constructed by Rippon and Stallard in [17, Example 6.1], in order to prove that there exists a 2-log-regular function of finite order which is not strongly logregular.

We will need the following result:

Lemma 5.2. Let $\phi$ and $\psi$ be real functions defined on $(0, \infty)$ with $\liminf _{t \rightarrow \infty} \phi(t)>$ 1 and such that

$$
\phi(t) \sim \psi(t), \quad \text { as } t \rightarrow \infty
$$

Let $\varepsilon \in(0,1)$. If for some $\varepsilon^{\prime} \in(0, \varepsilon)$, there exist $t_{0}>0$ and $k>1$ such that

$$
\phi\left(e^{k t}\right) \geq \exp \left(\frac{k}{\varepsilon^{\prime}} \phi(t)\right), \quad \text { for } t \geq t_{0}
$$

then there exists $t_{1}>0$ such that

Proof. Let

$$
\psi\left(e^{k t}\right) \geq \exp \left(\frac{k}{\varepsilon} \psi(t)\right), \quad \text { for } t \geq t_{1} .
$$

$$
\phi(t)=\psi(t)(1+\epsilon(t))
$$

where $\epsilon(t) \rightarrow 0$ as $t \rightarrow \infty$ and suppose that $\phi$ satisfies (5.8). Then

$$
\log \phi\left(t^{k}\right) \geq \frac{k}{\varepsilon^{\prime}} \phi(\log t), \quad \text { for } \log t \geq t_{0} .
$$

It follows from (5.9) and (5.10) that

$$
\log \psi\left(t^{k}\right)+\log \left(1+\epsilon\left(t^{k}\right)\right) \geq \frac{k}{\varepsilon^{\prime}} \psi(\log t)(1+\epsilon(\log t)), \quad \text { for } \log t \geq t_{0} .
$$


Hence, since $1+\epsilon(\log t) \rightarrow 1$ as $t \rightarrow \infty, \log \left(1+\epsilon\left(t^{k}\right)\right) \rightarrow 0$, as $t \rightarrow \infty, \varepsilon^{\prime} \in(0, \varepsilon)$, and $\lim \inf _{t \rightarrow \infty} \phi(t)>1$, there exists $t_{1}>0$, such that

$$
\log \left(\psi\left(t^{k}\right)\right) \geq \frac{k}{\varepsilon} \psi(\log t), \quad \text { for } t \geq t_{1},
$$

as claimed.

Example 5.1. There exists a transcendental entire function of finite order that is 2-log-regular but not strongly log-regular.

Proof. The main idea of the proof is to use a function $f$ constructed by Rippon and Stallard [17, Example 6.1], which has order zero and is not log-regular (and hence is not strongly log-regular) and show that $f$ is 2-log-regular.

In order to show that $f$ is 2-log-regular we need to show that, for any $\varepsilon>0$, there exist $r_{0}>0$ and $k>1$ such that

$$
\mu_{2, \varepsilon}\left(\exp \left(r^{k}\right)\right) \geq \exp \left(M(r)^{k}\right), \quad \text { for } r \geq r_{0},
$$

or equivalently,

$$
\log \left(M\left(\exp \left(r^{k}\right)\right) \geq M(r)^{k / \varepsilon}, \quad \text { for } r \geq r_{0} .\right.
$$

Hence, the condition we have to prove for $\psi(t)=\log M\left(e^{t}\right)$ is that, for any $\varepsilon \in(0,1)$, there exist $t_{1}>0$ and $k>1$ such that

$$
\psi(\exp (k t)) \geq \exp \left(\frac{k}{\varepsilon} \psi(t)\right), \quad \text { for } t \geq t_{1} .
$$

The function $f$ in [17, Example 6.1] was found by first constructing a real, increasing, convex function $\phi$ with certain properties and then using a result of Clunie and Kövari from [7] (see [17, Lemma 6.3]) in order to obtain a transcendental entire function $f$ such that $\psi(t)=\log M\left(e^{t}\right) \sim \phi(t)$.

Let $\mu(t)=\exp \left(t^{1 / 2}\right), t \geq 0$. Then take $t_{0}>1$ so large that $\exp \left(\frac{3}{4} t^{1 / 2}\right)>t$ for $t \geq t_{0}$, and define $t_{n}=\mu^{n}\left(t_{0}\right)$ and $k_{n}=t_{n+1}^{1 / 4}, n \geq 0$. Rippon and Stallard defined $\phi$ as follows:

$$
\phi(t)= \begin{cases}\mu_{n}(t), & t \in\left[t_{n+1} / k_{n}, t_{n+1}\right], \\ \mu(t), & \text { otherwise },\end{cases}
$$

where $\mu_{n}(t)$ denotes the linear function such that $\mu_{n}(t)=\mu(t)$ for $t=t_{n+1} / k_{n}=$ $t_{n+1}^{3 / 4}, t=t_{n+1}$.

We will first show that for any $\varepsilon^{\prime} \in(0,1)$, there exist $t_{0}>0$ and $k>1$ such that

$$
\phi(\exp (k t)) \geq \exp \left(\frac{k}{\varepsilon^{\prime}} \phi(t)\right), \quad \text { for } t \geq t_{0} .
$$

Let $\varepsilon^{\prime} \in(0,1)$ and $k>1$. When $\phi(t)=\mu(t)=\exp \left(t^{1 / 2}\right)$, we have $\phi(\exp (k t)) \geq \mu(\exp (k t))=\exp \left(\exp \left(\frac{1}{2} k t\right)\right) \geq \exp \left(\frac{k}{\varepsilon^{\prime}} \exp \left(t^{1 / 2}\right)\right)=\exp \left(\frac{k}{\varepsilon^{\prime}} \phi(t)\right)$, for $t$ large enough, and so (5.12) holds for these values of $t$.

Now suppose that $t \in\left[t_{n+1}^{3 / 4}, t_{n+1}\right]$, for some $n \in \mathbf{N}$. Then,

$$
\begin{aligned}
\phi(\exp (k t)) \geq \phi\left(\exp \left(k t_{n+1}^{3 / 4}\right)\right) & \geq \mu\left(\exp \left(k t_{n+1}^{3 / 4}\right)\right)=\exp \left(\exp \left(\frac{1}{2} k t_{n+1}^{3 / 4}\right)\right) \\
& \geq \exp \left(\frac{k}{\varepsilon^{\prime}} \exp \left(t_{n+1}^{1 / 2}\right)\right), \quad \text { for } t_{n+1} \text { large enough, } \\
& =\exp \left(\frac{k}{\varepsilon^{\prime}} \phi\left(t_{n+1}\right)\right) \geq \exp \left(\frac{k}{\varepsilon^{\prime}} \phi(t)\right),
\end{aligned}
$$


and hence (5.12) is satisfied.

Now, Lemma 5.2 implies that $\psi$ satisfies (5.11), which means that $f$ is 2-logregular.

\section{References}

[1] Anderson, J. M., and A. Hinkkanen: Unbounded domains of normality. - Proc. Amer. Math. Soc. 126, 1998, 3243-3252.

[2] Bergweiler, W.: Iteration of meromorphic functions. - Bull. Amer. Math. Soc. 29, 1993, $151-188$.

[3] Bergweiler, W., and A. Hinkkanen: On semiconjugation of entire functions. - Math. Proc. Cambridge Philos. Soc. 126, 1999, 565-574.

[4] Bergweiler, W., and B. KarpińsKa: On the Hausdorff dimension of the Julia set of a regularly growing entire function. - Math. Proc. Cambridge Philos. Soc. 148, 2010, 531-551.

[5] Bergweiler, W., B. Karpińska, and G. M. Stallard: The growth rate of an entire function and the Hausdorff dimension of its Julia set. - J. London Math. Soc. 80, 2009, 680-698.

[6] Bergweiler, W., and D. Nicks: Foundations for an iteration theory of entire quasiregular maps. - Israel J. Math. 201, 2014, 147-184.

[7] Clunie, J. G., and T. Kövari: On integral functions having prescribed asymptotic growth, II. - Canad. J. Math. 20, 1968, 7-20.

[8] Eremenko, A. E.: On the iteration of entire functions. - In: Dynamical systems and ergodic theory, Banach Center Publications 23, Polish Scientific Publishers, Warsaw, 1989, 339-345.

[9] Eremenko, A.E., and M. Yu. Lyubich: Dynamical properties of some classes of entire functions. - Ann. Inst. Fourier (Grenoble) 42, 1992, 989-1020.

[10] Evdoridou, V.: Fast escaping points of entire functions: a new regularity condition. - Math. Proc. Cambridge Philos. Soc. 160:1, 2016, 95-106.

[11] Martí-Pete, D.: The escaping set of transcendental self-maps of the punctured plane. Ergodic Theory and Dynam. Systems (to appear).

[12] Peter, J.: Hausdorff measure of escaping and Julia sets for bounded type functions of finite order. - Ergodic Theory and Dynam. Systems 33, 2013, 284-302.

[13] Rippon, P. J., and G. M. Stallard: Dimensions of Julia sets of meromorphic functions. - J. London Math. Soc. (2) 71, 2005, 669-683.

[14] Rippon, P. J., and G. M. Stallard: On questions of Fatou and Eremenko. - Proc. Amer. Math. Soc. 133, 2005, 1119-1126.

[15] Rippon, P. J., and G. M. Stallard: Functions of small growth with no unbounded Fatou components. - J. Anal. Math. 108, 2009, 61-86.

[16] Rippon, P. J., and G. M. Stallard: Fast escaping points of entire functions. - Proc. London Math. Soc. 105, 2012, 787-820.

[17] Rippon, P. J., and G. M. Stallard: Regularity and fast escaping points of entire functions. - Int. Math. Res. Not. IMRN 2014:19, 2014, 5203-5229.

[18] Rippon, P. J., and G. M. Stallard: Annular itineraries for entire functions. - Trans. Amer. Math. Soc. 367:1, 2015, 377-399.

[19] Rottenfusser, G., J. RÜCkert, L. Rempe, and D. Schleicher: Dynamic rays of boundedtype entire functions. - Ann. of Math. (2) 173, 2011, 77-125.

Received 25 July $2016 \bullet$ Accepted 20 February 2017 